\title{
A Comparative Study with Carvedilol and Ivabradine in Ischaemic Heart Disease Patients with Heart Failure
}

\author{
HARISUL HOQUE, KHURSHED AHMED, MOHAMMAD FAISAL IBN KABIR, NILUFAR FATEMA \\ Division of Heart failure, Rehabilitation and Preventive cardiology, Department of Cardiology, Bangabandhu Sheikh Mujib \\ Medical University (BSMMU), Shahbagh, Dhaka. \\ Address of Correspondence: Prof. Harisul Hoque, Head, Division of Heart failure, Rehabilitation and Preventive Cardiology, \\ Department of Cardiology, Bangabandhu Sheikh Mujib Medical University, Shahbagh, Dhaka. Email: harishoque@yahoo.com
}

University Heart Journal 2019; 15(2): 60-62

\section{Introduction:}

The leading cause of heart failure in developed countries is CAD. ${ }^{1}$ The prevalence of CAD and CAD related heart failure in developing countries is also increasing. The progression of the number of heart failure patients reflects the aggressive nature of the underlying CAD. Ischaemic cardiomyopathy (ICM) is the predominant form of heart failure related to CAD. Additional complications of CAD that may become superimposed on ischemic cardiomyopathy and precipitate heart failure are the development of LV aneurysm and mitral regurgitation caused by papillary muscle dysfunction. ${ }^{2}$ Elevated heart rate is associated with increased cardiovascular morbidity. The Ivabradine reduces heart rate without affecting cardiac contractility, and has been shown to be cardioprotective in the failing heart. Ivabradine also exerts some of its beneficial effects by decreasing cardiac proinflammatory cytokines and inhibiting peroxidants and collagen accumulation in atherosclerosis or congestive heart failure. The effects of ivabradine in the setting of acute viral myocarditis and on the cytokines, oxidative stress and cardiomyocyte apoptosis has shown a beneficially effect. Both ivabradine and carvedilol similarly and significantly reduced heart rate, attenuated myocardial lesions and improved the impairment of left ventricular function. In addition, ivabradine treatment as well as carvedilol treatment showed significant effects on altered myocardial cytokines with a decrease in the amount of plasma noradrenaline. Although beneficial effects of each of these two can be known from different studies. Without much knowledge regarding their precise position in the treatment of conditions like IHD with HF where the balance between optimum and excess is critical. As a result, to have a clear idea on their precise effects in symptomatic HF patients in ischemic setting practical demonstration is the key to enrich our experience and knowledge in the field of IHD related heart failure. This study is nothing but a way of piloting our darkness of unknowingness to the light of true understanding and knowingness.

\section{Materials and Methods:}

This pilot study was done in University Cardiac Centre, BSMMU from $1^{\text {st }}$ July, 2017 to $30^{\text {th }}$ June, 2018. 100 patients who will be diagnosed as a case of ischaemic heart disease with heart failure admitted to department of cardiology in BSMMU. 72 male patients and 28 female patients were included of 35 to 75 years of age. Fulfilling the ethical requirements detailed history, clinical examination and relevant investigation reports of all patients will be recorded in a pre-designed data collection sheet at the beginning of the study. Patients will be diagnosed as ICM patients by means of clinical evaluation and transthoracic echocardiography. Then, functionally patients will be classed according to NYHA class ${ }^{3}$ for heart failure and CCS class $^{3}$ for angina. After diagnosis and functional classification each patient will be put either to ivabradine or carvedilol group by lottery. 50 patients were given ivabradine treatment (Group-1) and another 50 patients were given carvedilol treatment (Group-2).

Each selected patient would undergo thorough history taking, clinical evaluation, echocardiography (TTE) and other relevant investigations during their stay in hospital and on discharge. Follow up of the patients will be done on $6^{\text {th }}$ and $12^{\text {th }}$ weeks. Patients' symptoms, functional classes and echocardiographic data (LV dimension and EF) will be recorded.

After collection, data was collected and organized. Then comparison and analysis of data (functional classes and LVEF) obtained from two groups of patients was done and results of the computation will be published. 


\section{Inclusion Criteria:}

Patient with IHD with HF which has been used to describe significantly impaired left ventricular function (LVEF $\leq 50$ percent) that is evidenced clinically and /or by noninvasive testing.

\section{Exclusion Criteria:}

Non-consenting patient.

\section{Statistical Analysis:}

Categorical data was expressed in percentage or number. Parametric data was expressed in mean + SD. Parametric data was evaluated by independent $t$ test and categorical data was evaluated by Chi square test. Significance are defined when $p$-value $<0.05$. Data analysis was conducted by SPSS 16.0 for windows software.

\section{Results:}

The patient distribution according to gender was 72 male patients and 28 female patients (Table I). The age average was determined as 56 \pm 2 . In Group-2, 37 (74\%) patients had showed reduced heart rate(HR) which is more than group1 where 28 patients $(56 \%)$ (Fig :1). Blood pressure (BP) was significantly decreased more in Group-1 but there was no effect on BP. Ivabradine can be use in shock patient as rate limiting drug as it has no effect on BP. Bronchospasm was evident in only carvidilol group in $6(12 \%)$ patients. $8(16 \%)$ patients in Group-1 developed oedema and worsening of heart failure symptoms but other patients of same group showed remarkable symptomatic improvement of HF. In group-2 patients didnot show any effect on HF symptom. In long term follow up of HF patients Group 1 showed less hospitalization that Group 2. After 6 months' treatment LVEF for the 50 patients of Carvedilol group (50\%; Group: A) improved by $(48.65 \% \pm 4.5)$ to $(51.32 \% \pm 4.2),(\mathrm{p}>0.05)$ and for the 50 patients of Ivabradine group (50\%; Group: B) $(48.2 \% \pm 4.4)$ to $(49 \% \pm 1.1),(p>0.05)$. There is significant improvement of EF in group-1.

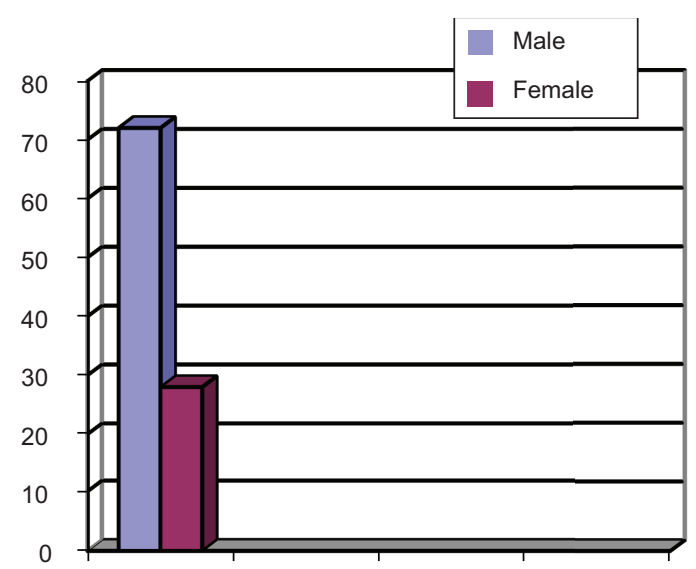

Fig.-1: Sex distribution of Heart failure in IHD patients

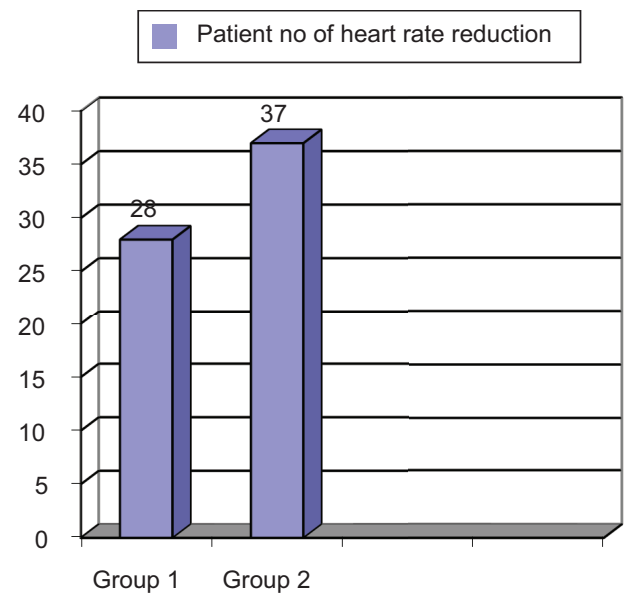

Fig.-2: Comparison on number of patients with Heart rate reduction between Group-1(Carvedilol) and Group2 (Ivabradine)

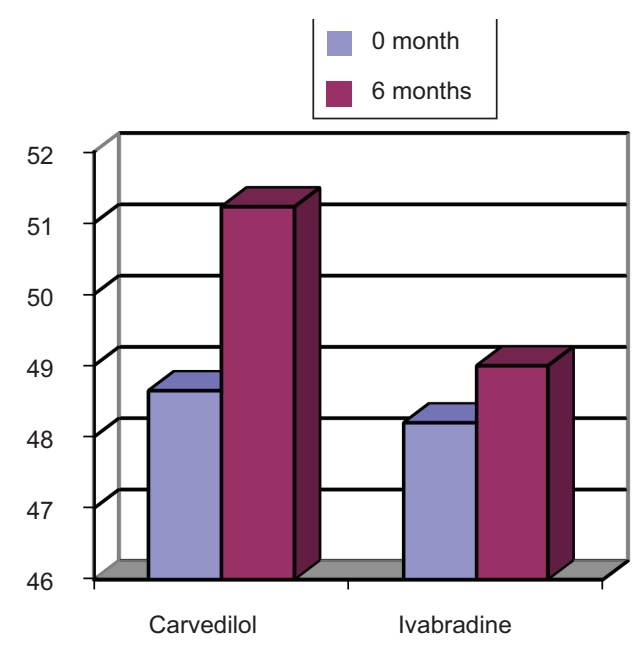

Fig.-3: Improvement of EF in Group 1 (Carvedilol) and Group 2 (Ivabradine)

\section{Discussion:}

In our study, we have compared the effects of Carvedilol and Ivabradine in Acute coronary syndrome patients with mild LV systolic dysfunction ( $\mathrm{LVEF} \leq 50 \%$ ). Improvement of EF were observed in Carvedilol group but Ivabradine group has shown no significant effect on the LVEF. In the SHIFT ${ }^{3}$ and BEAUTIFUL ${ }^{4}$ studies, Ivabradine was reported as having no adverse effects on the LVEF. The results of the BEAUTIFUL study have demonstrated that Ivabradine is a good choice for antianginal and antiischemic treatment, that it reduces the incidence of myocardial infarction and the need for coronary revascularization, and that it has a good tolerability profile when used in combination with other drugs. This study 
has also shown that Ivabradine use represents advancement in the treatment of ACS patients with heart failure with rates of $\geq 90$ beats per minute, and that the isolated decrease in heart rate caused by Ivabradine decreased the occurrence of Cardivascular events with optimal cardiovascular protective therapies. The results of this study are in parallel with the above mentioned studies. When ivabradine at a dose of $10 \mathrm{mg}$ /day compared with Carvedilol dose of $12.5 \mathrm{mg} /$ day, we observed that the efficacy of both drugs further increased, while the daily dose requirement and the patients' use of nitrate and trimetazidine decreased. Even at minimal levels, the daily dose of Ivabradine reduces the heart rate significantly. In our study, the effects of the Ivabradine and Carvedilol mono therapies on the respiratory system were evaluated. According to our study's results, Ivabradine has not demonstrated any effect that might lead to pulmonary dysfunction. It has been shown that Ivabradine had no adverse effect on the pulmonary functions of patients with COPD and pulmonary hypertension in a study. ${ }^{5}$ Our results have also demonstrated that Ivabradine can potentially be used as an antitachycardia agent in patients with COPD, bronchospasm and bronchial asthma. We observed that Carvedilol had minimal effect on pulmonary dysfunction. De Luca et al have conducted on 111 patients with EFs below 50\% described Ivabradine's effect in improving diastolic parameters on its own. ${ }^{6}$ Our results support the findings of the above mentioned study. To the best of our knowledge, our study is the first comparative study to be conducted in Bangladesh on Ivabradine and Carvedilol-using stable angina pectoris patients with a LVEF $\leq 50 \%$. In our study, we have compared the rates of hospitalization observed with mono therapies of Ivabradine and Carvedilol in ACS with HF patients with
LVEF 45 to $50 \%$. Among patients included into Group A and $\mathrm{B}$, no hospitalization was observed by the end of the six month. While no significant differences were noted between these two groups (Group A and B). These two drugs as monotherapy would provide a safer approach with regards to the side effect profile.

\section{Conclusion:}

Carvedilol showed significant improvement of HF symptoms in IHD patients both in short and long term. Ivabradine is a good choice for rate limiting in these sub groups of patients.

\section{References:}

1. Flaherty JD, Bax JJ, De Luca L, et al: Acute heart failure syndromes in patients with coronary artery disease early assessment and treatment. J Am Coll Cardiol 2009; 53:254.

2. Bonow RO, Mann DL, Zipes DP, Libby P, editors. Braunwald's heart disease. $10^{\text {th }}$ ed. India: Elsevier:2015.

3. Swedberg K, Komajda M, et al. Ivabradine and outcomes in chronic heart failure (SHIFT): A randomised placebocontrolled study. Lancet 2010; 376: 875-85.

4. Kim Fox, Ian Ford, P Gabriel Steg, et al. Ivabradine for patients with stable coronary artery disease and left-ventricular systolic dysfunction (BEAUTIFUL): A randomised, doubleblind, placebo-controlled trial. Lancet 2008; 372: 807-16.

5. Akhmetzianova ÉKh, Ga-nitdinova VV, Bakirov AB, Bogoroditskaia OA, Timershina IR. Effect of ivabradine on pulmonary hypertension in chronic obstructive pulmonary disease. Kardiologiia 2012; 52: 41-46.

6. De Luca G. Ývabradine and diastolic heart failure. Am Coll Cardiol. 2012; 59: E1009. Fox K, Garcia MA, Ardissino D, et al. Guidelines on the management of stable angina pectoris: Executive summary: The task force on the management of stable angina pectoris of the European Society of Cardiology. Eur Heart J. 2006; 27: 1341-81. 